\title{
How can we help women lose weight after child birth? Formative findings from the WeighWell weight management study
}

\author{
A. M. Craigie ${ }^{1}$, R. S. Barbour ${ }^{2}$, A. S. Anderson ${ }^{1}$ and the Weighwell team \\ ${ }^{1}$ Centre for Public Health Nutrition Research, University of Dundee, Dundee DD1 9SY, UK and ${ }^{2}$ School of Nursing and \\ Midwifery, University of Dundee, 11 Airlie Place, Dundee DD1 4HJ, UK
}

Pregnancy is a life stage where excess weight gain may occur and the post partum period is often characterised by weight retention, which often has negative implications for future health ${ }^{(1)}$. For the overweight or obese mother, weight loss is desirable for her own well-being, to meet the physical demands of parenting, to reduce the risk of obesity-related complications in further pregnancies ${ }^{(2)}$ and to promote future family health.

The WeighWell study is a scoping and feasibility study which aims to develop and implement a weight loss intervention for postpartum women living in areas of social deprivation, prior to undertaking a randomised controlled trial (RCT). The results of the formative work used to inform the design of the feasibility study protocol are described here.

Ten face-to-face interviews with key stakeholders working with the target group (midwives, health visitors and community support staff) were undertaken. The interviews explored diet and activity interventions, routes for communicating with the target group, and ways of obtaining optimal recruitment and retention rates. The interviews were complemented by five focus-group discussions with mothers $>1$ year post partum with a BMI over $25 \mathrm{~kg} / \mathrm{m}^{2}$ and living in areas of deprivation in Tayside and Sheffield. Discussions explored barriers and facilitators to adopting healthy diet and activity practices, routes to lifestyle change, structured and unstructured approaches, recruitment avenues and preferences for the intervention design and delivery.

The key findings from the formative work were:

- The post-partum period is perceived as a challenging time for weight loss. Barriers include body weight being a low priority, healthy food and exercise being perceived as expensive, a lack of time, energy, self-confidence or motivation and post-partum mental health problems.

- Motivators included the concept of 'dropping a dress size' as a goal, regular weight monitoring, personalisation and providing advice that could also benefit the baby and the rest of the family.

- A range of opportunities for recruiting the target group were identified e.g. via community groups, midwives and health visitors. Face-to-face contact was considered the most desirable.

- Suggested time for post-partum recruitment highlighted avoiding the initial 1-3 months and noting preferences and challenges for childcare (e.g. many women do not like using crèches).

- Preferences for group $v$. individualised support varied. Home-based exercise received mixed views, but positive experiences of new technology (e.g. Nintendo $\mathrm{Wii}^{\mathrm{TM}}$ ) were reported.

These findings have informed the design of the WeighWell feasibility study currently being undertaken in women between 6 and 18 months post partum in Tayside. The intervention incorporates personalised advice over a 3-month period. This is provided via monthly counselling sessions, goal setting according to personally identified goals, weight monitoring and literature advising on low-cost lifestyle changes that can be adopted by the whole family and undertaken in the home environment.

Funding was provided by the Medical Research Council.

1. Villamor E \& Cnattingius S (2006) Interpregnancy weight change and risk of adverse pregnancy outcomes: a population-based study. Lancet 368, $1164-1170$.

2. Galtier-Dereure F, Boegner C \& Bringer J (2000) Obesity and pregnancy: complications and cost. Am J Clin Nutr 71 1242S-1248S. 